PROCEEDINGS OF THE

AMERICAN MATHEMATICAL SOCIETY

Volume 133, Number 3, Pages 745-749

S 0002-9939(04)07718-4

Article electronically published on October 21, 2004

\title{
SEMILINEAR ELLIPTIC EQUATIONS AND FIXED POINTS
}

\author{
CLEON S. BARROSO
}

(Communicated by David S. Tartakoff)

\begin{abstract}
In this paper, we deal with a class of semilinear elliptic equations in a bounded domain $\Omega \subset \mathbb{R}^{N}, N \geq 3$, with $C^{1,1}$ boundary. Using a new fixed point result of the Krasnoselskii type for the sum of two operators, an existence principle of strong solutions is proved. We give two examples where the nonlinearity can be critical.
\end{abstract}

\section{INTRODUCTION}

Consider the boundary value problem for a semilinear elliptic equation:

$\left(P_{\lambda, \mu}\right) \quad\left\{\begin{array}{l}-\Delta u+\lambda u=f(x, u, \mu) \quad \text { in } \Omega, \\ u=0 \quad \text { on } \partial \Omega,\end{array}\right.$

where $\Omega$ is a bounded domain in $\mathbb{R}^{N}(N \geq 3)$ with $C^{1,1}$ boundary, $\Delta$ is the standard Laplace operator, $\lambda$ is a parameter close to 0 , and $f: \Omega \times \mathbb{R} \times \mathbb{R}^{+} \rightarrow \mathbb{R}$ is a Carathéodory function. Elliptic equations such as $\left(\overline{P_{\lambda, \mu}}\right)$ have been studied extensively for several years (see [1, 2, 3, 10, and the references therein). According to the behaviour of $f(\cdot, u)$, topological methods may come to be more appropriate to the solvability of $\left(\mid P_{\lambda, \mu}\right)$ than variational tools, especially when $f(\cdot, u)$ has critical growth due to the lack of compactness of Sobolev embedding.

In this paper, we study the problem $\left(\overline{P_{\lambda, \mu}}\right)$ by using fixed point methods. A new version of Krasnoselskii's fixed point theorem for the sum of two operators given by the author [6] is used to establish an existence principle for this problem. Here we will be interested in the existence of a strong solution to $\left(P_{\lambda, \mu}\right)$, that is, a function $u \in W^{2,2}(\Omega) \cap W_{0}^{1,2}(\Omega)$ such that $-\Delta u(x)+\lambda u(x)=f(x, u(x))$ for almost every $x \in \Omega$ and fulfilling the boundary condition.

A special case of $\left(\overline{P_{\lambda, \mu}}\right)$ is the problem

$\left(Q_{\lambda, \mu}\right) \quad\left\{\begin{array}{l}-\Delta u+\lambda u=\mu u^{p}+h(x) \quad \text { in } \Omega, \\ u=0 \quad \text { on } \partial \Omega,\end{array}\right.$

where $p>1, \mu>0$ and $h \in L^{2}(\Omega)$. When $\lambda=0$ and $\mu=1$ this problem relates to an open question (cf. [1, pg. 124]): For any $h$ in $L^{2}(\Omega)$, does $\left(Q_{\lambda, \mu}\right)$ have infinitely many solutions? Bahri $[8$ has given a partial answer. He proved for $p \in] 2,2^{*}[$ that there is an open dense set of $h$ in $W^{-1,2}(\Omega)$ for which $Q_{\lambda, \mu}$ possesses an

Received by the editors September 25, 2003.

2000 Mathematics Subject Classification. Primary 35J25; Secondary 47H10.

Key words and phrases. Semilinear elliptic equations, fixed point theorem, Krasnoselskii.

This research was supported by Capes, Brazil. 
infinite number of distinct weak solutions. In [9] the authors showed that there exists $1<p_{N}<(N+2) /(N-2)$ such that for any $h \in L^{2}(\Omega)$ and $p \in\left(1, p_{N}\right)$ the previous equation admits infinitely many distinct solutions. Here, we show for any $h \in L^{2}(\Omega), h \neq \equiv 0$, and $p>1$ if $N=3$, or $1<p \leq N /(N-4)$ if $N>4$, or still $1<p \leq 2$ if $N=4$, that there exists a positive constant $\mu_{p, h}$ such that $Q_{\lambda, \mu}$ has a nontrivial strong solution if $\mu \in\left(0, \mu_{p, h}\right]$. Furthermore, if $h \in L^{2}(\Omega)$ satisfies $h \geq 0$ almost everywhere in $\Omega$, then the solution is positive. Notice that if $N>4$, then $(N+2) /(N-2)<N /(N-4)$. Thus, the technique used in [8, 9] cannot be applied to solve $\left(Q_{\lambda, \mu}\right)$ when $p=2^{*}$ and $N=3$, or $p_{N} \leq p \leq N /(N-4)$ if $N>4$. Moreover, when $N=4$ we have that $p_{4}<2$, so that $\left(1, p_{4}\right) \subseteq(1,2]$.

The paper is organized as follows. The main result and some preliminaries are presented in Section 2. In Section 3 we prove the main result. In the last section we study the problem $\left(Q_{\lambda, \mu}\right)$ and another special case of $\left(P_{\lambda, \mu}\right)$ related to an eigenvalue problem.

\section{MAin RESUlt}

Throughout this paper $\Omega$ denotes a bounded domain in $\mathbb{R}^{N}(N \geq 3)$ with $C^{1,1}$ boundary. In this section, let $E$ denote the space $W^{2,2}(\Omega) \cap W_{0}^{1,2}(\Omega)$, where $W^{2,2}(\Omega)$ is the usual Sobolev space and $W_{0}^{1,2}(\Omega)$ is the closure of $C_{0}^{\infty}(\Omega)$ in the norm $\|u\|_{1,2, \Omega}$. It is well known that the mapping $\phi: u \mapsto-\Delta u$ is one-to-one from $E$ onto $L^{2}(\Omega)$. Moreover, there is a constant $C>0$ such that

$$
\|u\|_{2,2} \leq C\|\Delta u\|_{2},
$$

for all $u \in E$; see [7] Lemma 9.17]. Now write $\lambda^{*}=\left\|\phi^{-1}\right\|_{\mathcal{L}^{2}(\Omega)}^{-1}$.

Given a Carathéodory function $f: \Omega \times \mathbb{R} \times \mathbb{R}^{+} \rightarrow \mathbb{R}$, the assumptions below will be assumed in what follows:

$\left(\mathcal{H}_{1}\right)$ there exists $\mu>0$ such that $N_{f_{\mu}}\left(\phi^{-1}\right)\left(B_{R}\right) \subseteq B_{R}$, for some $R>0$;

$\left(\mathcal{H}_{2}\right)$ there exists $\mu>0$ such that $N_{f_{\mu}}\left(\phi^{-1}\right)^{+}\left(B_{R}\right) \subseteq B_{R}$, for some $R>0$;

$\left(\mathcal{H}_{3}\right) f_{\mu}(x, u)=f(x, u, \mu) \geq 0$ if $u \geq 0$

$\left(\mathcal{H}_{4}\right) p>1$ if $N=3$, or $1<p \leq N /(N-4)$ if $N>4$, or still $1<p \leq 2$ if $N=4$, where $N_{f_{\mu}}(u)=f(\cdot, u, \mu)\left(\right.$ resp. $\left.N_{f_{\mu}}(u)^{+}=f_{\mu}\left(\cdot, u^{+}\right)\right)$and $B_{R}=B_{R}(0) \subseteq L^{2}(\Omega)$.

In this paper we prove the following theorem.

Theorem 1. Let $0 \leq \lambda \leq \lambda^{*}$. Suppose $f: \Omega \times \mathbb{R} \times \mathbb{R}^{+} \rightarrow \mathbb{R}$ is a Carathéodory function. Then the following hold:

(a) $\left(P_{\lambda, \mu}\right)$ has a strong solution if $\left(\mathcal{H}_{1}\right)$ is fulfilled.

(b) $\left(P_{\lambda, \mu}\right)$ has a nonnegative strong solution if $\left(\mathcal{H}_{2}\right)-\left(\mathcal{H}_{3}\right)$ are verified.

This improves upon the recent work in [6], Theorem 4.1, which instead of $\left(\mathcal{H}_{1}\right)$ requires the assumption $N_{f} \phi^{-1}\left(B_{R C}\right) \subseteq B_{R}$ with $C>0$ satisfying (2.1) and some $R>0$. As we will see in Section 4, in some concrete situations the assumption $\left(\mathcal{H}_{1}\right)$, or $\left(\mathcal{H}_{2}\right)$, seems to be more effective that this condition.

The proof of Theorem 1 is based on the following asymptotic version of Krasnoselskii's fixed point theorem for the sum of two operators given by the author in [6].

Theorem 2. Let $X$ be a reflexive space and $B \in \mathcal{L}(X)$ with $\left\|B^{p}\right\| \leq 1, p \geq 1$, a dissipative operator on $X$. If $A: X \rightarrow X$ is a weakly continuous mapping such that $A\left(B_{R}\right) \subseteq B_{R}$, for some $R>0$, then there exists $y \in B_{R}$ such that $A y+B y=y$. 


\section{Proof of Theorem 1}

The proof is similar to that of Theorem 4.1 of [6], and we include it here for the sake of completeness. Let $B$ be the linear operator in $L^{2}(\Omega)$ given by $B u=$ $-\lambda \phi^{-1}(u)$ with $0<\lambda \leq \lambda^{*}$. Next, define $A: B_{R} \rightarrow L^{2}(\Omega)$ by

$$
A(u)= \begin{cases}N_{f_{\mu}}\left(\phi^{-1}(u)\right) & \text { for the case }(a), \\ N_{f_{\mu}}\left(\phi^{-1}(u)^{+}\right) & \text {for the case }(b) .\end{cases}
$$

Claim I. $B$ is a dissipative operator in $L^{2}(\Omega)$ with $\|B\| \leq 1$.

Proof of Claim I. Indeed, by the choice of $\lambda$ we have $\|B\| \leq 1$. Now, because of Green's first identity we have $(\phi(u), u)_{L^{2}(\Omega)}=-\int_{\Omega} \Delta u \cdot u d x=\int_{\Omega}|\nabla u|^{2} d x \geq 0$, for all $u \in E$. Using this we see that $(B u, u)_{L^{2}(\Omega)} \leq 0$, for all $u \in L^{2}(\Omega)$, which implies that $B$ is a dissipative operator in $L^{2}(\Omega)$. This proves Claim I.

Claim II. $A$ is weakly continuous in $B_{R}$.

Proof of Claim II. It is sufficient to show the claim for the item (b); see [6]. Clearly $A$ is well defined and by $\left(\mathcal{H}_{2}\right)$ maps $B_{R}$ into itself. Now let $\left\{u_{n}\right\}$ be a sequence in $B_{R}$ such that $u_{n} \rightarrow u$ in $B_{R}$. Then, we have $\phi^{-1}\left(u_{n_{j}}\right) \rightarrow \phi^{-1}(u)$ in $W^{2,2}(\Omega)$ (see 7. Lemma 9.17, pg. 242]). Since the embedding $W^{2,2}(\Omega) \hookrightarrow L^{2}(\Omega)$ is compact, $\phi^{-1}\left(u_{n_{j}}\right) \rightarrow \phi^{-1}(u)$ in $L^{2}(\Omega)$. Now, Vainberg's theorem [4] gives $\phi^{-1}\left(u_{n}\right)^{+} \rightarrow$ $\phi^{-1}(u)^{+}$and $N_{f_{\mu}}\left(\phi^{-1}\left(u_{n}\right)^{+}\right) \rightarrow N_{f_{\mu}}\left(\phi^{-1}(u)^{+}\right)$in $L^{2}(\Omega)$. Hence $N_{f_{\mu}}\left(\phi^{-1}\left(u_{n}\right)^{+}\right) \rightarrow$ $N_{f_{\mu}}\left(\phi^{-1}(u)^{+}\right)$in $L^{2}(\Omega)$. This shows that $A$ is weakly sequentially continuous in $B_{R}$. On the other hand, since $L^{2}(\Omega)$ is reflexive and separable, $\left(B_{R}, w\right)$ is a bounded and metrizable topological space, where $w$ denotes the weak topology of $L^{2}(\Omega)$. Owing to theorem $A 6(b)$ of [5, pg. 370] we conclude that $A$ is weakly continuous in $B_{R}$. This ends the proof of the claim.

Hence, Theorem 2 with $X=L^{2}(\Omega)$ and $A, B$ as above guarantees the existence of a fixed point of $A+B$ in $B_{R}$, say $u$. By using the assumption $\left(\mathcal{H}_{3}\right)$ we may apply the weak maximum principle as stated for instance in [1] to conclude that $v=\phi^{-1}(u)$ is a strong solution to $\overline{P_{\lambda, \mu}}$, with $v(x) \geq 0$ for almost every $x \in \Omega$. The $\lambda=0$ case follows from Claim II together with the Schauder fixed point theorem for locally convex spaces. The proof of Theorem 1 is thereby complete.

\section{Special Cases}

Theorem 1 has a variety of simple and practical special cases. As a first example, it can be used to solve the problem $\left(Q_{\lambda, \mu}\right)$ mentioned in the introduction.

Theorem 3. Assume $\left(\mathcal{H}_{4}\right)$ and $\lambda \geq 0$ as above. Then, for any $h \in L^{2}(\Omega), h \not \equiv 0$, there is $\mu_{p, h}>0$ such that the problem $Q_{\lambda, \mu}$ has a nontrivial strong solution if $\mu \in\left(0, \mu_{p, h}\right]$. Moreover, if $h \geq 0$ almost everywhere in $\Omega$, then the solution is positive.

Proof. First, let us consider the cases $N=3$ and $N>4$. Thanks to [7, (7.30) pg. $158]$ we know that the imbeddings $W^{2,2}(\Omega) \hookrightarrow C^{0}(\Omega)$ and $W^{2,2}(\Omega) \hookrightarrow L^{\frac{2 N}{N-4}}(\Omega)$ are continuous for $N=3$ and $N>4$, respectively. Combining this with $\left(\mathcal{H}_{4}\right)$ and (2.1) yields

$$
\left\|\phi^{-1}(u)\right\|_{2 p} \leq \gamma\|u\|_{2}
$$


for all $u \in L^{2}(\Omega)$ and some $\gamma=\gamma_{p}(\Omega)>0$. Let $h(x)$ be as above and define $\mu_{p, h}=\frac{1}{\left(2^{p} \gamma\|h\|_{2}^{p-1}\right)}$. Next, fixing $\mu \in\left(0, \mu_{p, h}\right]$ and setting $\beta=(2 \mu \gamma)^{1 /(p-1)}$ we define

$$
f(x, u, \mu)=\frac{u^{p}}{2 \gamma}+\beta h(x) .
$$

We are now going to verify the condition $\left(\mathcal{H}_{1}\right)$. From 4.1) we have

$$
\left\|N_{f_{\mu}}\left(\phi^{-1}(u)\right)\right\|_{2} \leq \frac{1}{2}\|u\|_{2}^{p}+\beta\|h\|_{2},
$$

for all $u \in L^{2}(\Omega)$. Since $\beta=(2 \mu \gamma)^{1 /(p-1)} \leq 1 / 2$, by (4.2) it is immediate to verify that the condition $\left(\mathcal{H}_{1}\right)$ holds for $R=1$. Then, Theorem 1 (a) yields a strong solution to $\left(\overline{P_{\lambda, \mu}}\right)$, say $u$. At last, it is easy to check that $v=(u / \beta)$ solves $\left(Q_{\lambda, \mu}\right)$.

In a similar way, the estimate (4.2) remains valid for $\left\|N_{f_{\mu}}\left(\phi^{-1}(u)^{+}\right)\right\|_{2}$, and hence the condition $\left(\mathcal{H}_{3}\right)$ holds for $R=1$, too. Thus, if $h(x) \geq 0$ for almost every $x \in \Omega$ we can apply Theorem 1 (b) to conclude that $u \geq 0$. Using again the weak maximum principle it follows that either $u \equiv 0$ or $u>0$. Since $h \not \equiv 0$ we get $u>0$.

When $N=4$, we can use the continuous imbeddings $W^{2,2}(\Omega) \hookrightarrow W^{1,2}(\Omega) \hookrightarrow$ $L^{4}(\Omega)$ together with (2.1) to give again (4.1), if $1<p \leq 2$. In particular, in exactly the same way as above we get similar conclusions for this case. This completes the proof.

Our second application refers to the following eigenvalue problem:

$$
\left\{\begin{array}{l}
-\Delta u+\lambda u=\mu g(x, u) \quad \text { in } \Omega, \\
u=0 \quad \text { on } \partial \Omega
\end{array}\right.
$$

where $g: \Omega \times \mathbb{R} \rightarrow \mathbb{R}$ is a Carathéodory function satisfying

$$
|g(x, u)| \leq a|u|^{p}+b(x),
$$

where $a>0, b \in L^{2}(\Omega)$ and $p>1$.

Theorem 4. Assume $\left(\mathcal{H}_{4}\right)$ and $0 \leq \lambda \leq \lambda^{*}$. Then, there is $\mu^{*}$ such that (4.3) has at least one strong solution if $\mu \in\left(0, \mu^{*}\right]$.

Proof. In order to give a strong solution for the problem (4.3) by using Theorem 1 we define $f(x, u, \mu)=\mu g(x, u), \mu>0$. In view of (4.1) we then derive

$$
\left\|N_{f_{\mu}}\left(\phi^{-1}(u)\right)\right\|_{2} \leq \mu\left(a \gamma\|u\|_{2}^{p}+\|b\|_{2}\right),
$$

for all $u \in L^{2}(\Omega)$. Thus, if $u \in B_{1} \subseteq L^{2}(\Omega)$ and $\mu^{*}=\frac{1}{\left(a \gamma+\|b\|_{2}\right)}$, it follows from (4.4) that the condition $\left(\mathcal{H}_{1}\right)$ holds for any $\mu \in\left(0, \mu^{*}\right]$. Theorem[1 $(a)$ now ensures that (4.3) has a strong solution.

\section{ACKNOWLEDGEMENT}

The author expresses his sincere gratitude to the referee for helpful comments. He also thanks Dr. José Fábio Montenegro for his encouragement. 


\section{REFERENCES}

[1] M. Struwe, Variational Methods, Springer, Berlin, 1990. MR1078018 (92b:49002)

[2] Paul H. Rabinowitz, Minimax methods in critical point theory with applications to differential equations. CBMS Regional Conference Series Math. 65, Amer. Math. Soc., Providence (1986). MR.0845785 (87j:58024)

[3] M. Willem, Minimax Theorems, Birkhäuser, Basel, 1996. MR1400007 (97h:58037)

[4] M.M. Vainberg, Variational methods for the study of non-linear operators. Holden-Day, San Francisco, CA (1964). MR0176364 (31:638)

[5] W. Rudin, Functional Analysis, McGraw-Hill, 1973. MR0365062 (51:1315)

[6] Cleon S. Barroso, Krasnoselskii's fixed point theorem for weakly continuous maps, Nonlinear Anal. 55 (2003) 25-31. MR2001629 (2004f:47084)

[7] D. Gilbarg, N.S. Trundiger, Elliptic Partial Differential Equations of Second Order, Springer, Berlin, 1983. MR0737190 (86c:35035)

[8] A. Bahri, Topological results on a certain class of functionals and applications, J. Funct. Anal. 41 (1981), 397-427. MR0619960 (84c:58017)

[9] A. Bahri and H. Berestycki, A perturbation method in critical point theory and applications, Trans. Amer. Math. Soc. 267 (1981), 1-32. MR0621969 (82j:35059)

[10] Jin, Zhiren, Multiple solutions for a class of semilinear elliptic equations, Proc. Amer. Math. Soc. 125 (1997), 3659-3667. MR1443158 (98h:35078)

Departamento de Matematica, Universidade Federal do Ceará, Campus do Pici, Bl. 914, Fortaleza-Ce, 60455-760, BraziL

E-mail address: cleonbar@mat.ufc.br 